\title{
Barrier bucket experiment at the AGS
}

\author{
M. Fujieda, Y. Iwashita, and A. Noda \\ Nuclear Science Research Facility, Institute for Chemical Research, Kyoto University, Gokanosho, Uji 611, Japan \\ Y. Mori, C. Ohmori, and Y. Sato* \\ KEK-Tanashi, 3-2-1 Midori-cho, Tanashi 188, Japan \\ M. Yoshii \\ KEK, 1-1 Oho, Tsukuba 305, Japan
}

M. Blaskiewicz, J. M. Brennan, T. Roser, K. S. Smith, R. Spitz, and A. Zaltsmann

Brookhaven National Laboratory, Upton, New York 11973

(Received 26 August 1999; published 30 December 1999)

\begin{abstract}
A barrier bucket experiment with two dedicated barrier cavities was performed at the Brookhaven AGS. One of the barrier cavities was a magnetic alloy (MA)-loaded cavity and the other was a ferriteloaded cavity. They generated a single sine wave with a peak voltage of $40 \mathrm{kV}$ at a repetition rate of $351 \mathrm{kHz}$. A barrier rf system was established with these cavities and five bunches from the AGS booster were accumulated. A total of $3 \times 10^{13}$ protons were stored without beam loss, and were successfully rebunched and accelerated. The longitudinal emittance growth was observed during accumulation by the barrier bucket, the blowup factor of which was about 3. The longitudinal mismatch between the rf bucket and the beam bunch was the main reason for the emittance growth. The potential distortions by beam loading of the ferrite cavity and the overshooting voltage of the MA cavity disturbed the smooth debunching.
\end{abstract}

PACS numbers: 29.20.Lq, 29.27.Ac

\section{INTRODUCTION}

Slow beam loss caused by a space-charge-induced tune shift during the injection and accumulation processes in the case of ordinary bucket-to-bucket transfer is one of the biggest problems in a high-intensity proton synchrotron. The tune shift is in inverse proportion to the bunching factor, $B_{f}=\lambda_{\text {average }} / \lambda_{\text {peak }}$. Here, $\lambda_{\text {average }}$ and $\lambda_{\text {peak }}$ are the average and peak line density of the beam, respectively. In order to reduce the tune shift, it is necessary to decrease the peak line density of the beam.

One possibility, which makes the beam distribution flat for decreasing the peak line density, is a higher harmonic rf system. For example, in a second-harmonic rf system, $B_{f}$ could be about 0.5 . In another way, a barrier bucket (BB) scheme [1-3], $B_{f}$ could become almost 1 by using a flat potential. This would also enable the accumulation of more bunches than the harmonic number of the ring, and may increase the number of injections up to the limitation of the momentum acceptance. Thus, the BB scheme is expected to be an efficient way to increase the beam intensity of a proton synchrotron.

The last BB experiment was carried out to show the efficiency for increasing the intensity at the AGS [2]. The AGS booster was then operated with harmonic

\footnotetext{
*Also at Japan Steel Works Co. Ltd.
}

number $h=2$, and the AGS was operated with harmonic number $h=8$. Two dedicated barrier cavities were used. They generated a single sine wave of $2 \mathrm{MHz}$ triggered at the revolution frequency. A peak voltage of $12 \mathrm{kV}$ per cavity allowed a beam to be stored with a momentum spread of \pm 0.002 . Although six injections of one bunch accumulated $3 \times 10^{13}$ protons, the operation of adiabatic debunching was not performed and the longitudinal emittance was allowed to grow. In a test aimed at conserving the emittance, multiple injections of one bunch were performed and a fast instability was observed during debunching due to a small momentum spread. In those studies, the longitudinal emittance grew because the moving barrier could not be turned off adiabatically and a quantitative measurement of the emittance growth was not carried out.

The present BB experiment $[4,5]$ was performed during the 1998 high-intensity proton run at the AGS [6] under the Japan-U.S. collaboration of high-energy physics. Two dedicated barrier cavities were used in this experiment. One of them, developed by KEK [7], involved magnetic alloy (MA) cores which can carry out adiabatic turning off. Because of its low $Q$ value $(Q \sim 0.6)$, the MA cavity can generate a single sine wave with much less rf power than that of a high- $Q$ cavity. The other cavity is a ferrite-loaded type, which was modified from the ordinary accelerating cavity by BNL. The AGS booster has been operated with harmonic number $h=1$, and the AGS has run with harmonic number $h=6$. This allows 
six injections of one bunch per AGS cycle and increases the intensity per bunch. The momentum spreads of the bunches were higher than before. A peak barrier voltage of $40 \mathrm{kV}$ has been supplied to accumulate $10^{14}$ protons by two dedicated barrier cavities, allowing a beam with a momentum spread of \pm 0.004 to be stored. An MA cavity was first tested with a high-intensity beam. It was also the first trial to rebunch and accelerate an accumulated beam by applying BB. A detailed study concerning the adiabatic condition has also been performed. This paper describes the experimental results.

\section{BARRIER BUCKET SCHEME}

In the $\mathrm{BB}$ scheme, two barriers are required because one of them must create a flat rf bucket and the other has to perform barrier gymnastics. At first, they form long and short rf buckets in longitudinal phase space, as shown in Fig. 1(a). After a bunch is injected into the shorter bucket, the phase of a barrier is swept, while the other is fixed [Fig. 1(b)]. Thus, the bunch spreads in the extending bucket. This process is called "adiabatic debunching." After the bunch becomes sufficiently long, the moving barrier is turned off [Fig. 1(c)]. The first bunch spreads, except for the phase gap of the fixed barrier. The barrier is turned on at the same phase as the fixed barrier and is moved. This makes an empty bucket for the next bunch injection [Fig. 1(d)]. After the next injection, the barrier is moved again. When the momentum spreads of the first and second bunches become equal, the moving barrier is turned off [Fig. 1(e)],

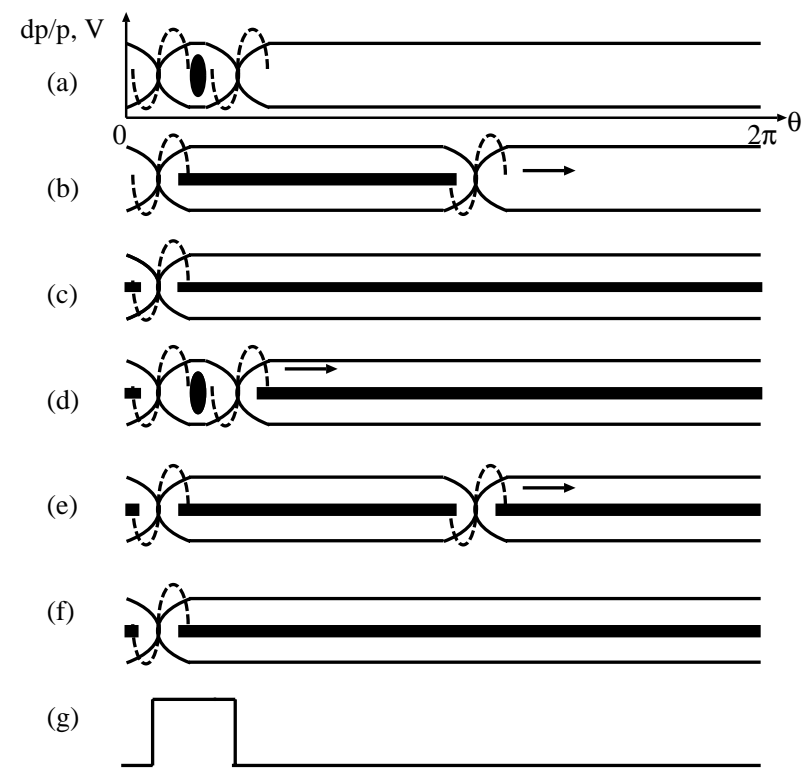

FIG. 1. Barrier bucket scheme. The horizontal and vertical coordinates are the rf phase and the momentum spread (solid line) or the rf voltage (dotted line), respectively. The upright ellipse means a bunch. The thick lines are the debunched beam; $(\mathrm{g})$ is the injection kicker pulse. and these bunches merge [Fig. 1(f)]. It is possible to repeat these three processes (adiabatic debunching, "merging," and "spacing") until the bucket is filled up. The number of injections is independent of the harmonic number of the ring.

It is necessary to perform these processes adiabatically in order to conserve the longitudinal emittance. Let us assume that the phase of the barrier voltage is moved $\Delta \phi$ per turn from the synchronous phase. The revolution period and frequency are set at $T_{\text {rev }}$ and $\omega_{\text {rev }} / 2 \pi$, respectively. The speed $(\dot{\Phi})$ is given by

$$
\dot{\Phi}=\frac{\Delta \phi}{T_{\text {rev }}} \text {. }
$$

A particle obtains a momentum deviation through an interaction with the moving barrier. The deviation, $\left(\frac{d p}{p}\right)_{\text {barrier }}$, is given by the phase equation, and is written as

$$
\left|\left(\frac{d p}{p}\right)_{\text {barrier }}\right|=\left|\frac{\dot{\Phi}}{\omega_{\text {rev }} \eta}\right|,
$$

where $\eta$ is the slippage factor. The adiabatic condition requires that the speed $(\dot{\Phi})$ should be much smaller than the drift rate $\left(\dot{\Phi}_{\text {drift }}\right)$,

$$
|\dot{\Phi}| \ll\left|\dot{\Phi}_{\mathrm{drift}}\right|=\omega_{\mathrm{rev}}|\eta|\left(\frac{d p}{p}\right)_{\max },
$$

where $\left(\frac{d p}{p}\right)_{\max }$ is the maximum momentum spread of the beam.

\section{EXPERIMENTAL SETUP}

\section{A. Barrier cavities}

Two barriers were necessary to perform barrier gymnastics. A peak voltage of $40 \mathrm{kV}$ with a period of $500 \mathrm{~ns}$ was required for the accumulation of $10^{14}$ protons in the AGS. The barriers were generated at a revolution frequency of $351 \mathrm{kHz}$ by two dedicated barrier cavities. One of them was an MA-loaded cavity developed by KEK; the other was a ferrite-loaded cavity, which was modified from the ordinary accelerating cavity by BNL.

The MA cores have the following characteristics: low $Q$, high inductance, stability for magnetic field strength, and high Curie temperature.

The MA cavity does not need a tuning loop because the frequency characteristic is sufficiently broad. We do not have to be concerned with a temperature rise in the cores, and thus the cooling system can be simple. A gradient acceleration field as high as $50 \mathrm{kV} / \mathrm{m}[8,9]$ has been achieved because of the stability against a strong $\mathrm{rf}$ magnetic field. The low $Q$ value enables us to generate a barrier voltage with less rf power, which can be explained as follows.

The cavity is assumed to be a parallel LCR circuit. The necessary current to generate a barrier voltage is 
expressed by

$$
I(t)=\frac{V(t)}{R}+\frac{1}{L} \int V\left(t^{\prime}\right) d t^{\prime}+C \frac{d V(t)}{d t}= \begin{cases}\frac{V_{0} \sin \omega t}{R}+\frac{V_{0}}{\omega L}+V_{0} \cos \omega t\left(\omega C-\frac{1}{\omega L}\right), & 0 \leq \omega t \leq 2 \pi \\ 0, & \text { otherwise }\end{cases}
$$

For the single sine wave used here,

$$
V(t)= \begin{cases}V_{0} \sin \omega t, & 0 \leq \omega t \leq 2 \pi \\ 0, & \text { otherwise }\end{cases}
$$

the required current on resonance is

$$
I(t)= \begin{cases}\frac{V_{0}}{R}(\sin \omega t+Q), & 0 \leq \omega t \leq 2 \pi, \\ 0, & \text { otherwise. }\end{cases}
$$

The necessary currents to generate a barrier for different $Q$ values of 0.6 and 3 are shown in Fig. 2. It is assumed that the value of $\frac{V_{0}}{R}$ is 10 . The current can be divided into the dc offset part, $\frac{V_{0} Q}{R}$, and the rf amplitude part, $\frac{V_{0} \sin \omega t}{R}$. The offset $\frac{V_{0} Q}{R}$ depends on the $Q$ value. Thus, one can see that a lower $Q$ value could enable us to generate a barrier voltage with less rf power. The waveform becomes like a sine wave in the case of a low $Q$, while it becomes like a square pulse in the case of a high $Q$. If a negative current is required to generate a barrier, a single-ended tube cannot be adopted. Therefore, a push-pull amplifier is suitable to drive a low- $Q$ cavity. Although the current waveform has been assumed to be a square in a paper [10], a bipolar waveform is necessary for a low- $Q$ cavity. The waveform (6) includes many harmonics. If the necessary currents for their harmonics are provided to a cavity, a perfect single sine wave is generated for any $Q$ value.

The parameters of the barrier cavities are given in Table I.

\section{B. Barrier setup}

The major parameters of the AGS are listed in Table II.

A BB experiment has occasionally been performed to obtain pulses from normal cycles for physics users. The

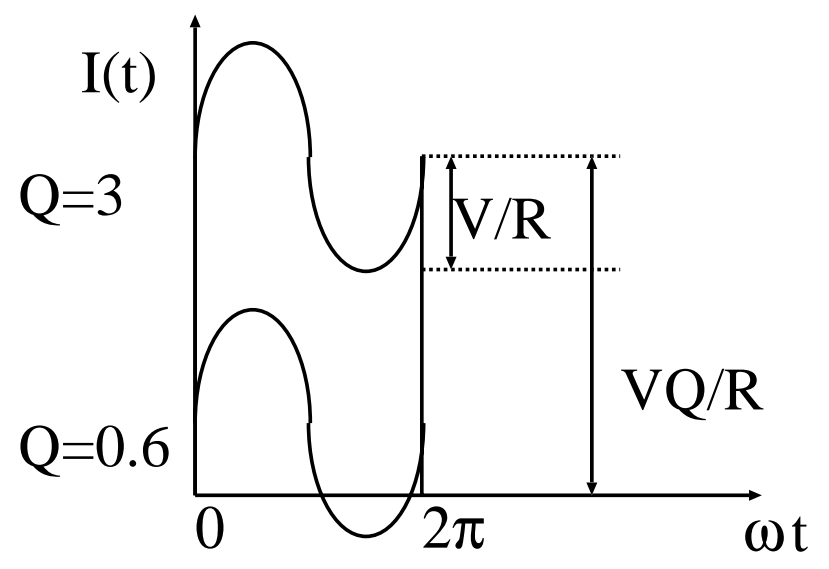

FIG. 2. Necessary peak currents for a barrier. The values of $V / R$ are fixed at 10 .
AGS booster was operated with a harmonic number of 1 , while the AGS was operated with a harmonic number of 6 during a high-intensity proton run. In the usual cycle, six bunches from the booster were transferred to the AGS. The number of transfers is not limited by the harmonic number for a BB scheme. Up to a five-bunch transfer was allowed, because a beam should be debunched sufficiently for rebunching and acceleration within an accumulation period where the start time of $\dot{B}$ is limited.

A barrier rf system using two dedicated barrier cavities was established. The frequency sweep and adiabatic turning off of the barriers have been performed as follows. The trigger signals at the revolution frequency were supplied to drive the cavities. One of their phases was swept by a counterphasing module, the patterns of which were easily edited by a computer. The amplitude-modulation function was generated in the same way. Amplitude modulation for the barrier generated by the ferrite cavity was not performed. The grid voltage was generated by combining a square pulse with a single sine wave. For a high-voltage pulse, fast field-effect transistor switches were used so as to step up the grid voltage from -500 to $-100 \mathrm{~V}$ during the barrier period. A broadband amplifier generated a single sine wave. Since both amplitudes of the square pulse and the single sine wave in the grid voltage must be controlled at the same time, a changeable dc supply was necessary. Therefore, only amplitude modulation for the barrier generated by the MA cavity was performed by modulating the amplitude of the input voltage at the grid. The MA cavity generated a moving barrier, while the ferrite cavity made a fixed barrier.

\section{Beam-loading compensation of the MA cavity}

The $R / Q$ of the MA cavity is so large that any beam loading must be compensated. The induced voltage can be canceled perfectly if the beam-monitor signal is

TABLE I. Barrier cavities parameters.

\begin{tabular}{lcc}
\hline \hline & MA cavity & Ferrite cavity \\
\hline The number of gaps & 4 & 4 \\
Total rf voltage $(\mathrm{kV})$ & 40 & 40 \\
Tube power $(\mathrm{kW})$ & $30 \times 2$ & 600 \\
Tube operation & class B & class AB \\
& push-pull & single ended \\
$Q$ value & 0.6 & 30 \\
$R / Q$ per cavity $(\mathrm{k} \Omega)$ & 6.0 & 0.72 \\
Resonant frequency $(\mathrm{MHz})$ & 1.5 & 2.6 \\
Beam loading correction & feed forward & none \\
\hline \hline
\end{tabular}


TABLE II. AGS parameters.

\begin{tabular}{lc}
\hline \hline Booster repetition rate & $7.5 \mathrm{~Hz}$ \\
Injection energy & $1.9 \mathrm{GeV}$ \\
Revolution frequency & $351 \mathrm{kHz}$ \\
$\gamma_{t}$ & 8.5 \\
AGS cycle (fast extraction) & $2.5 \mathrm{~s}$ \\
$\quad$ (slow extraction) & $5.1 \mathrm{~s}$ \\
\hline \hline
\end{tabular}

added to a broadband amplifier. A feed-forward scheme [11-13] is more practical than a feed-back scheme. Thus, a simplified feed-forward system was adopted to compensate for any beam loading.

The induced voltage in the low- $Q$ cavity includes as many Fourier components as those of the beam signal. Therefore, the frequency characteristic is important to know for making a beam-loading compensation system. The impedance-matched range of the grid circuit in the rf amplifier was higher than $1 \mathrm{MHz}$, and the frequency responses of the beam monitor and cables were not flat over the range where there were Fourier components of the beam. The feed-forward path was divided in order to make their responses equal. A schematic of the feedforward system is shown in Fig. 3.

The beam signal was picked up by a wall current monitor. The signal was split into three paths and filtered. The filters were low pass, band pass, and high pass, which passed $h=1, h=2$, and $h \geq 3$ Fourier components, respectively. A harmonic number of 1 means a revolution frequency of $351 \mathrm{kHz}$. Each phase and amplitude were adjusted independently. The feed-forward signal was

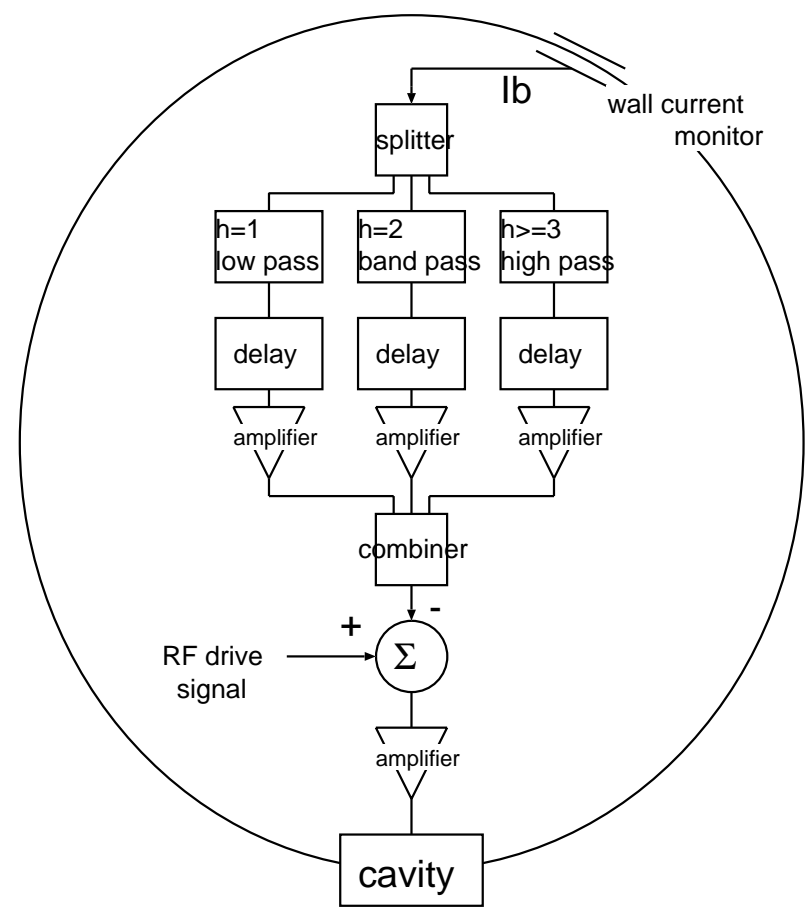

FIG. 3. Schematic view of the feed-forward system.
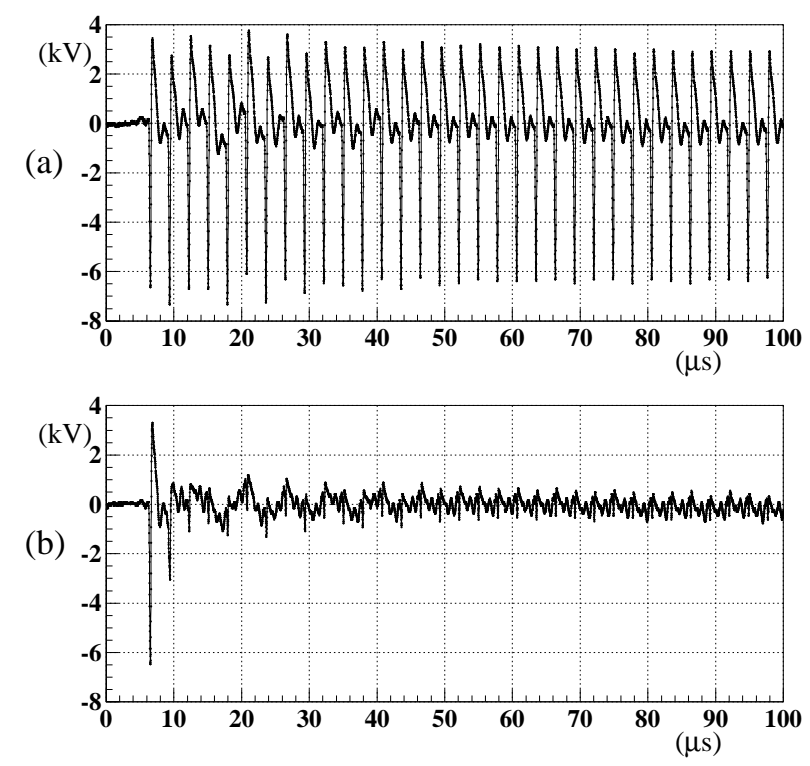

FIG. 4. Induced voltages: (a) without feed forward and (b) with feed forward. The horizontal and vertical coordinates are the time and the voltage per gap, respectively. The beam intensity was $8.2 \times 10^{12}$ protons per bunch and one bunch was circulating.

delayed so as to arrive at the cavity after one revolution period from the beam passage. This was due to the fact that the feed-forward path was longer than the beam traveling time from the monitor to the cavity.

The induced voltage and the impedance per gap with or without feed forward are shown in Figs. 4 and 5,

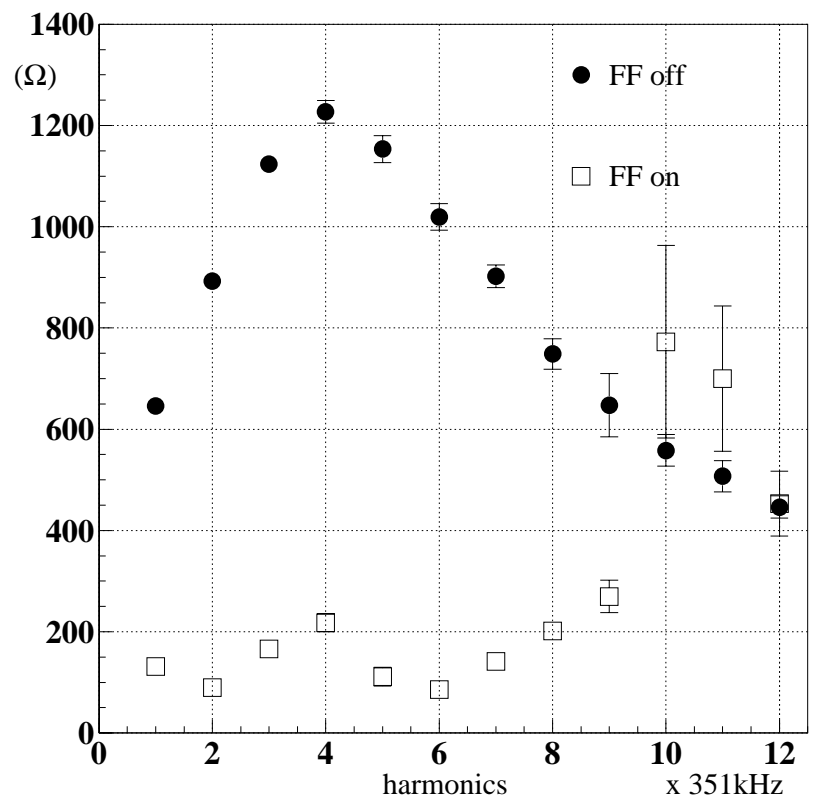

FIG. 5. Impedance per gap. The horizontal axis is the harmonics of a revolution frequency of $351 \mathrm{kHz}$. The black circles and the white squares are the impedance without and with feed forward, respectively. The error bars show statistical errors only. 
respectively. The impedance is given by

$$
Z(\omega)=\frac{V(\omega)}{I(\omega)},
$$

where $V(\omega)$ and $I(\omega)$ were obtained from the fastFourier-transform algorithm of the gap-voltage monitor signal and the wall-current monitor signal, respectively.

The induced voltage was effectively decreased by $1 / 9$. The rising time of the system was fast and the voltage was dumped in three turns. The impedance in the range between harmonic numbers of 1 and 8 became smaller than $200 \Omega$. The feed-forward system was not effective for harmonics of $h \geq 10$. Once the parameters of the system were optimized, they did not drift. The system worked stably for different beam conditions, for example, the intensity and bunch width. They were due to the stability of the MA cavity against a temperature rise or beam loading. It seems that a feed-forward scheme is suitable to compensate any beam loading of the MA cavity.

\section{Overshooting voltage cancellation of the MA cavity}

The barrier voltage generated by the MA cavity had an overshooting part. A bump was formed in the rf potential. This bump caught the beam and prevented it from debunching smoothly. The overshooting voltage was induced because the drive amplifier could not generate the same waveform of the current as in Eq. (6). Since an MA cavity can be driven by an arbitrary function, because of its broadband impedance, it is possible to improve the waveform by optimizing the drive signal.

Figure 6 shows the barrier voltages of the MA cavity. Waveform [Fig. 6(a)] was supplied in the BB experiment. An improved waveform [Fig. 6(b)] was achieved by a drive-signal modification at the end of the experiment, which involved a mixture of a few harmonics. In the high-gradient cavity [8], the overshooting voltage disappeared upon adding harmonics of 1 and 2 to the drive signal. It will be possible to cancel the overshooting voltage by applying the same scheme to the MA cavity.

\section{EXPERIMENTAL RESULTS}

\section{A. Spacing}

The first injected bunch circulates while spreading out over the ring, except for the phase gap of the fixed barrier at the second injection time. If a complete coasting beam is disturbed by the injection kicker, the kicked portion is lost. Therefore, the barriers should make a wide empty
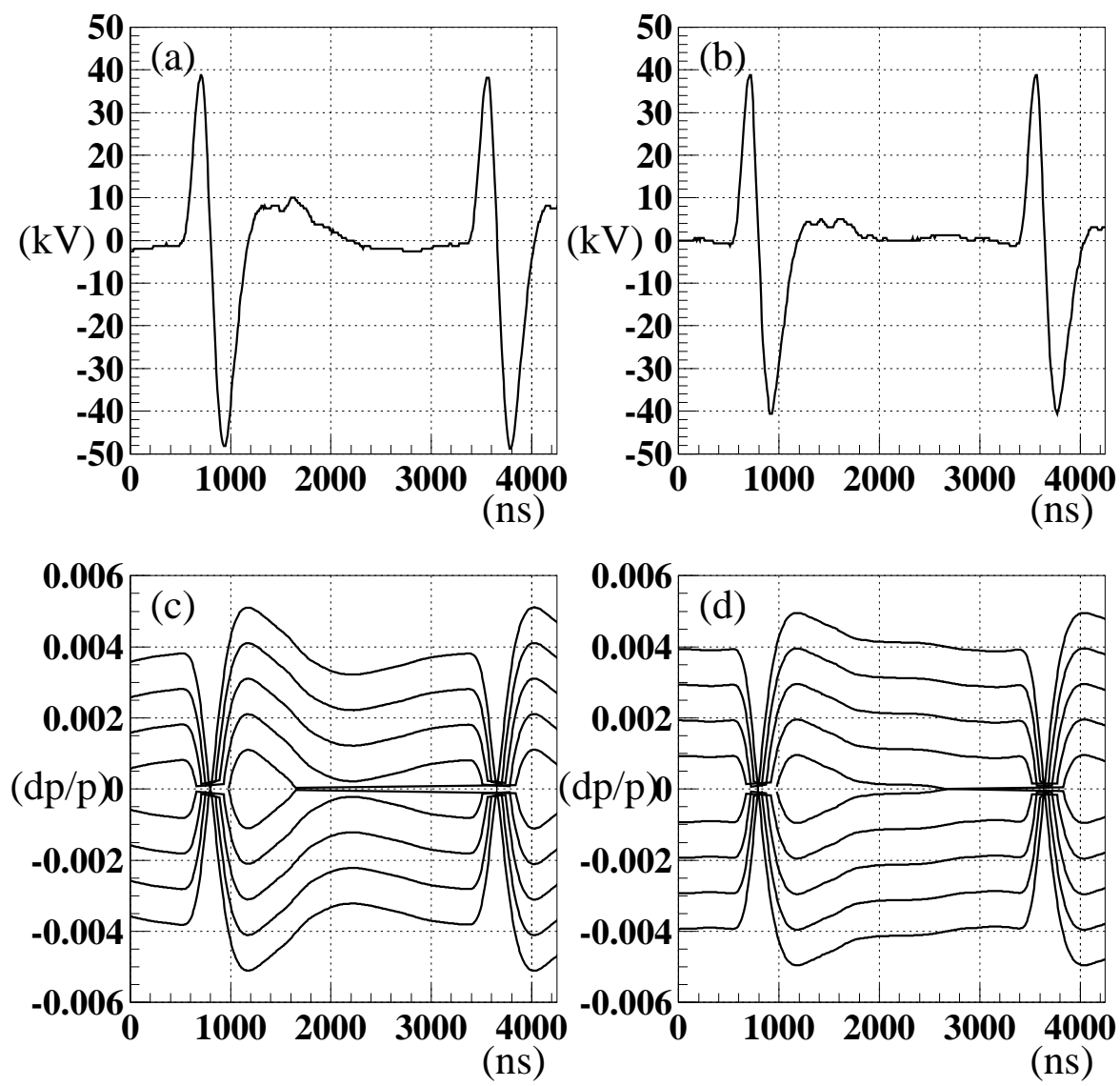

FIG. 6. Gap voltages (a),(b) and the rf buckets (c),(d). Waveform (a) was used in the experiment, which forms the rf bucket (c). The waveform (b) and the bucket (d) were improved. 


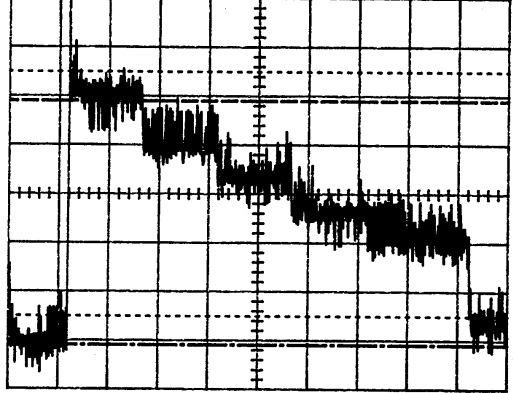

without barriers

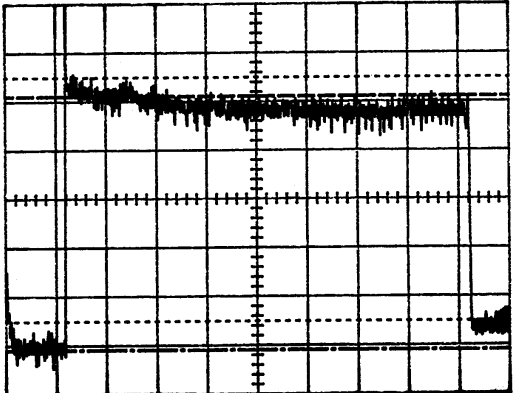

$0.1 \mathrm{~s} / \mathrm{div}$.

FIG. 7. Slow intensity monitor signals when the injection kicker was fired five times. The horizontal axis is time with $0.1 \mathrm{~s} /$ division. The vertical axis is $50 \mathrm{mV} /$ division. One bunch was injected and the intensity was $1.9 \times 10^{12}$ protons. Left: without barriers; right: phase gap of $97^{\circ}$ was made by barriers to avoid beam loss.

bucket in order to avoid any loss, as shown in Fig. 1(d). Figure $1(\mathrm{~g})$ shows the injection kicker pulse. A bunch is injected into an empty bucket. The initial phase gap between the barriers was optimized while observing the beam loss. There was no beam loss by the kicker when the phase gap was wider than $97^{\circ}$, which was consistent with the kicker pulse width. Figure 7 shows the slow intensity monitor signals when the injection kicker was fired five times after one bunch injection. While a part of the beam was lost in the left-hand case of no barrier, the beam survived in the right-hand case with barriers. The phase gap used to avoid any beam loss was wider than the typical bunch width of $24^{\circ}$, which caused a longitudinal phase-space mismatch between the rf bucket and the bunch.

\section{B. Adiabatic debunching measurement}

The adiabatic debunching, merging, and spacing processes in barrier gymnastics must be performed adiabatically so as to conserve the longitudinal emittance. The emittance was measured while varying the sweep speed of the moving barrier to see whether the adiabatic condition was satisfied or not. The gaps of the accelerating cavities were short circuited during the measurement. Since the measurement was performed with a single-bunch transfer, the initial phase gap was set at $77^{\circ}$ so as to suppress any longitudinal phase-space mismatch. As soon as the phase gap between the barriers was spread from $77^{\circ}$ to $315^{\circ}$, the gaps of the barrier cavities were short circuited. The momentum spread should have decreased by $\frac{77^{\circ}}{315^{\circ}}$ after adiabatic debunching. It was measured by the Schottky signal to determine the longitudinal emittance $100 \mathrm{~ms}$ after the timing of a gap short. The initial momentum spread was changed by the longitudinal quadrupole bunch-shape oscillation at extraction in the booster, which was controlled by amplitude modulation of the rf voltage. Therefore, the longitudinal emittance of bunches was fixed. In the case of amplitude modulation being present, the measurement was performed with initial momentum spreads of \pm 0.0044 and \pm 0.003 , while an initial momentum spread of \pm 0.0034 was used for a measurement without any amplitude modulation. The barrier experiments were usually performed without the presence of amplitude modulation. The results are shown in Fig. 8. The initial momentum spread was derived from the ordinary debunching, in which during the measurements the gaps of the barrier cavities were short circuited and the momentum spread was measured after debunching for $200 \mathrm{~ms}$.

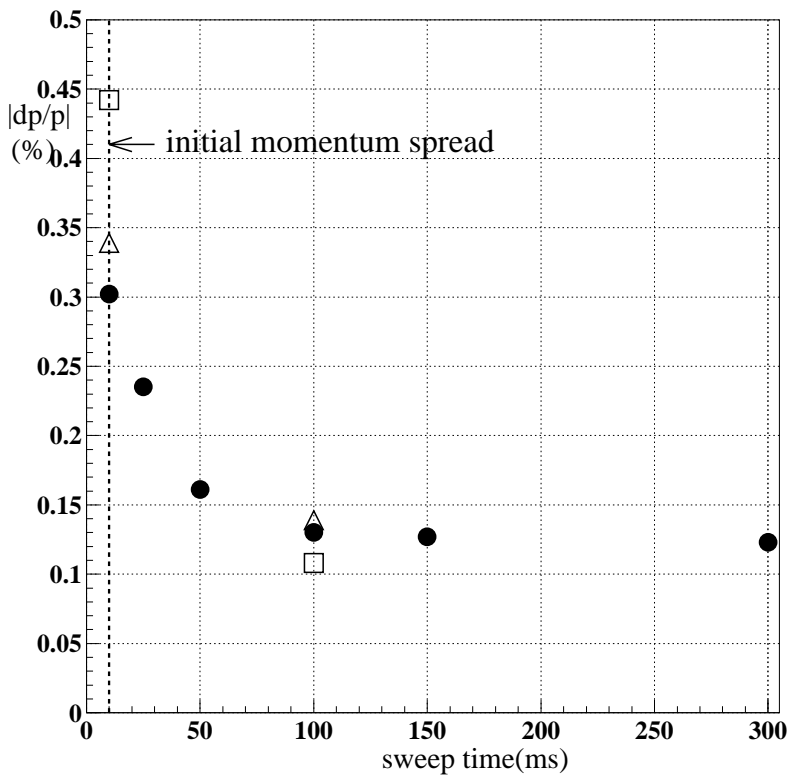

FIG. 8. Momentum spread against the sweep time. The markers on the dotted line show their initial $d p / p$. The different markers show the different initial $d p / p$. The initial $d p / p$ of the black circle is 0.003 . Those of the white triangle and square are 0.0034 and 0.0044 , respectively. The beam intensity was about $7 \times 10^{12}$ protons/bunch. 
The minimum momentum spreads were achieved in the case of a sweep speed slower than $100 \mathrm{~ms}$. Here, the maximum value of the initial momentum spread is assumed to be 0.003 and the sweep speed is set at $100 \mathrm{~ms}$. Their parameters are applied to Eq. (3). The sweep speed and the drift rate of the beam are written as

$$
\begin{aligned}
\dot{\Phi} & =4.2 \times 10(\mathrm{rad} / \mathrm{s}), \\
\dot{\Phi}_{\text {drift }} & =6.3 \times 10^{2}(\mathrm{rad} / \mathrm{s}) .
\end{aligned}
$$

If the barrier is swept as slowly as about one-tenth of the drift rate, the momentum spread is minimized and the adiabatic condition seems to be satisfied. The differences in the momentum spreads against the sweep time were consistent with the deviation, $\left(\frac{d p}{p}\right)_{\text {barrier }}$, derived from Eq. (2).

The momentum spread decreased by about $25 \%$ when its initial value was \pm 0.0044 . As for the initial values of \pm 0.0034 and \pm 0.003 , the momentum spreads decreased by about $40 \%$. Those values were larger than those based on the spread phase gap. The reason is discussed later in Sec. V B.

\section{Multiple transfers}

The barrier gymnastics for multiple transfers were established. In the merging process, the sweep range of the moving barrier was at first set geometrically. The phase of the moving barrier was rotated by $360^{\circ}$ for the first bunch. After the second bunch was injected, it was rotated by $180^{\circ}$ so as to equalize the momentum spread of the stored and freshly injected bunch. After the third, fourth, and fifth injections it was swept by $120^{\circ}, 90^{\circ}$, and $72^{\circ}$, respectively. Then, a fine adjustment was performed while observing the beam loss. Spacing was carried out in about $50 \mathrm{~ms}$. It took about $80 \mathrm{~ms}$ to perform adiabatic debunching. The adiabatic turning off of the moving barrier took about $10 \mathrm{~ms}$. Five injections of one bunch were successful. The phase and amplitude-modulation patterns of the moving barrier in five-bunch transfers are shown in Fig. 9.

A slow beam loss during accumulation was observed in the case of the ordinary bucket-to-bucket transfer in the AGS. A stacking beam without any loss was successfully performed with the barriers. No evidence was found that showed an instability during accumulation. This might have been due to the larger momentum spread compared to that in the last experiment. Figure 10 shows a mountain-range plot of the wall-current monitor during the accumulation of five bunches. The first trace was started $34 \mathrm{~ms}$ after the first injection.

\section{Rebunch and acceleration}

Rebunching and acceleration of the beam accumulated by the barriers were tried for the first time. After the last injection from the booster, the beam spread out over the ring, except for the phase gap at the fixed barrier. After the fixed barrier was snapped off, the gap was filled up. The gaps of the accelerating cavities were opened after the beam debunched sufficiently. Their voltages were increased linearly. The recaptured bunch widths were observed while changing the ramping time with a singlebunch transfer, which produced longitudinal emittances, as shown in Table III. A bunch width of $23^{\circ}$ corresponded to an emittance of $4.0 \mathrm{eV} \mathrm{s} /$ bunch, while the initial emittance, which was the value just after injection into the AGS, was $2.9 \mathrm{eV} \mathrm{s}$. It was found that the ramping time had to be slower than about $100 \mathrm{~ms}$ so as to avoid any longitudinal emittance growth, in which case there was no capture loss.

The longitudinal emittance was measured just before acceleration. The emittance of the beam rebunched by a harmonic number of 6 was about $7.0 \mathrm{eV} \mathrm{s}$ per bunch. The blowup factor, which is the ratio between the actual emittance and the initial emittance, becomes $(7.0 \times$ $6) /(2.9 \times 5)=2.9$. It was found that the emittance growth depended on the phase gap between the barriers and the gymnastics.

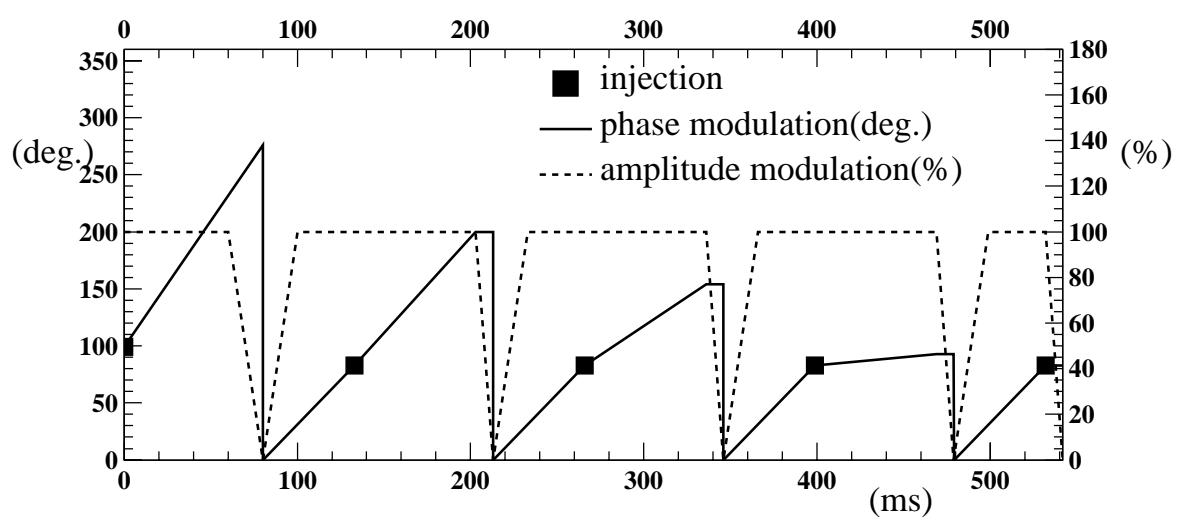

FIG. 9. Phase and amplitude modulation patterns for a moving barrier. The horizontal axis is the time from the first injection. The vertical axes are degree (left) and \% (right). The solid and dotted line are phase-modulation and amplitude-modulation patterns, respectively. The solid squares show the timings of injections. The phase of $0^{\circ}$ corresponds to that of the fixed barrier. 


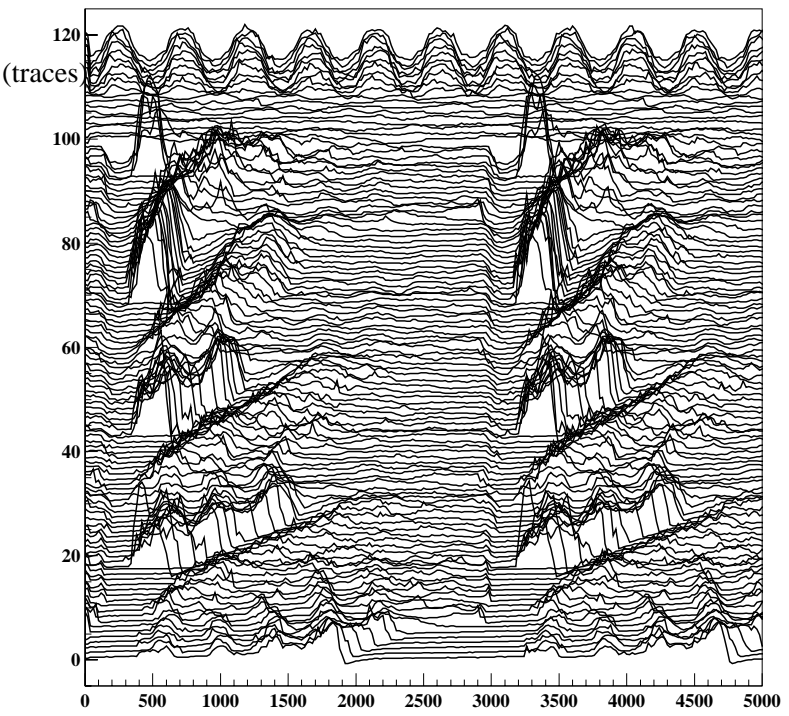

(ns)

FIG. 10. Mountain-range plot of the wall-current monitor. The interval of their traces is $5.8 \mathrm{~ms}$. The horizontal axis is the $\mathrm{rf}$ phase. The period of one revolution is $2850 \mathrm{~ns}$.

Acceleration was performed by the accelerating cavities. The slow intensity monitor signal in the low beam intensity is shown in Fig. 11 . A total of $3 \times 10^{13}$ protons were accumulated and accelerated. The intensity of the first bunch from the booster was $6 \times 10^{12}$ protons. A few losses were observed during acceleration. There was beam loss of about $10 \% \sim 20 \%$ at the transition gamma jump because of the longitudinal emittance. In order to reduce this loss, the emittance should be decreased.

\section{DISCUSSIONS}

Longitudinal emittance growth during five-bunch transfers was observed in the $\mathrm{BB}$ experiment. There are several possibilities which might have caused longitudinal emittance growth in the process. One of them involves a longitudinal mismatch between the rf bucket and the beam bunch. In order to avoid beam loss due to the injection kicker, the phase gap between the barriers was set to be wider than the typical bunch width. Therefore, it induced a longitudinal mismatch, which was unavoidable. Another possibility is a potential distortion, which would be caused by beam loading of the ferrite cavity and the overshooting voltage of the MA cavity.

TABLE III. Captured bunch widths for various ramping times.

\begin{tabular}{lccc}
\hline \hline & & Ramping time (ms) \\
& 50 & 135 & 270 \\
\hline Bunch width (degree) & 27 & 23 & 23 \\
\hline \hline
\end{tabular}

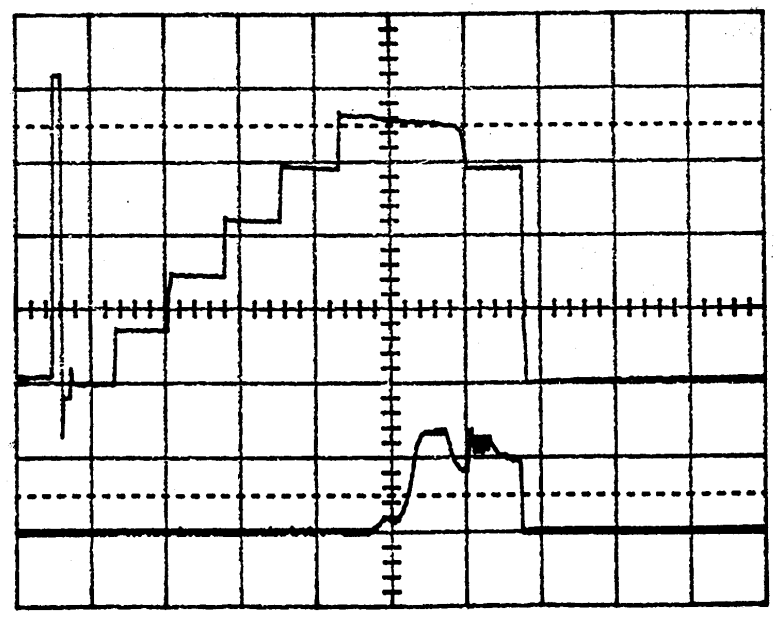

$200 \mathrm{~ms} / \mathrm{div}$

FIG. 11. Slow intensity monitor signal. The horizontal axis is time and its unit is $200 \mathrm{~ms} /$ division. The upper signal is the slow intensity monitor signal. The lower signal is the envelope of the accelerating voltage. A total of $1.3 \times 10^{13}$ protons were accumulated.

In order to clarify the causes of the longitudinal emittance growth, simulations of five-bunch transfers were performed using a multiparticle tracking code. In this section, the simulation results are presented. The simulation took only the longitudinal motion into account and included the effects of space charge and induced voltage at the rf cavity. A thousand macroparticles per bunch were used in the simulation. The actual barrier waveform could be applied. The bunch signal $I(t)$ in the time domain was Fourier transformed into the frequency domain $I(\omega)$. The wake voltage and space-charge voltage were derived from the cavity impedance and the spacecharge impedance, multiplying by $I(\omega)$, respectively. Their voltages $V(\omega)$ were inverse-Fourier transformed again into the time domain. The cavity impedance of the LCR resonator type was used while assuming the parameters given in Table I.

\section{A. Longitudinal mismatch}

A longitudinal mismatch between the rf bucket and the beam bunch causes filamentation and emittance growth in longitudinal phase space. In the BB experiment, a phase gap of more than $770 \mathrm{~ns}$ between the barriers was necessary to avoid any beam loss due to the injection kicker pulse. Since the phase gap formed by the barriers makes a flat potential, which is wider than the typical bunch width, as shown in Fig. 12, a phase-space mismatch is unavoidable. In order to estimate the blowup factor due to a longitudinal mismatch, simulations of five-bunch transfers were carried out under the same condition as presented in Fig. 9. Two of the single sine waves were used 

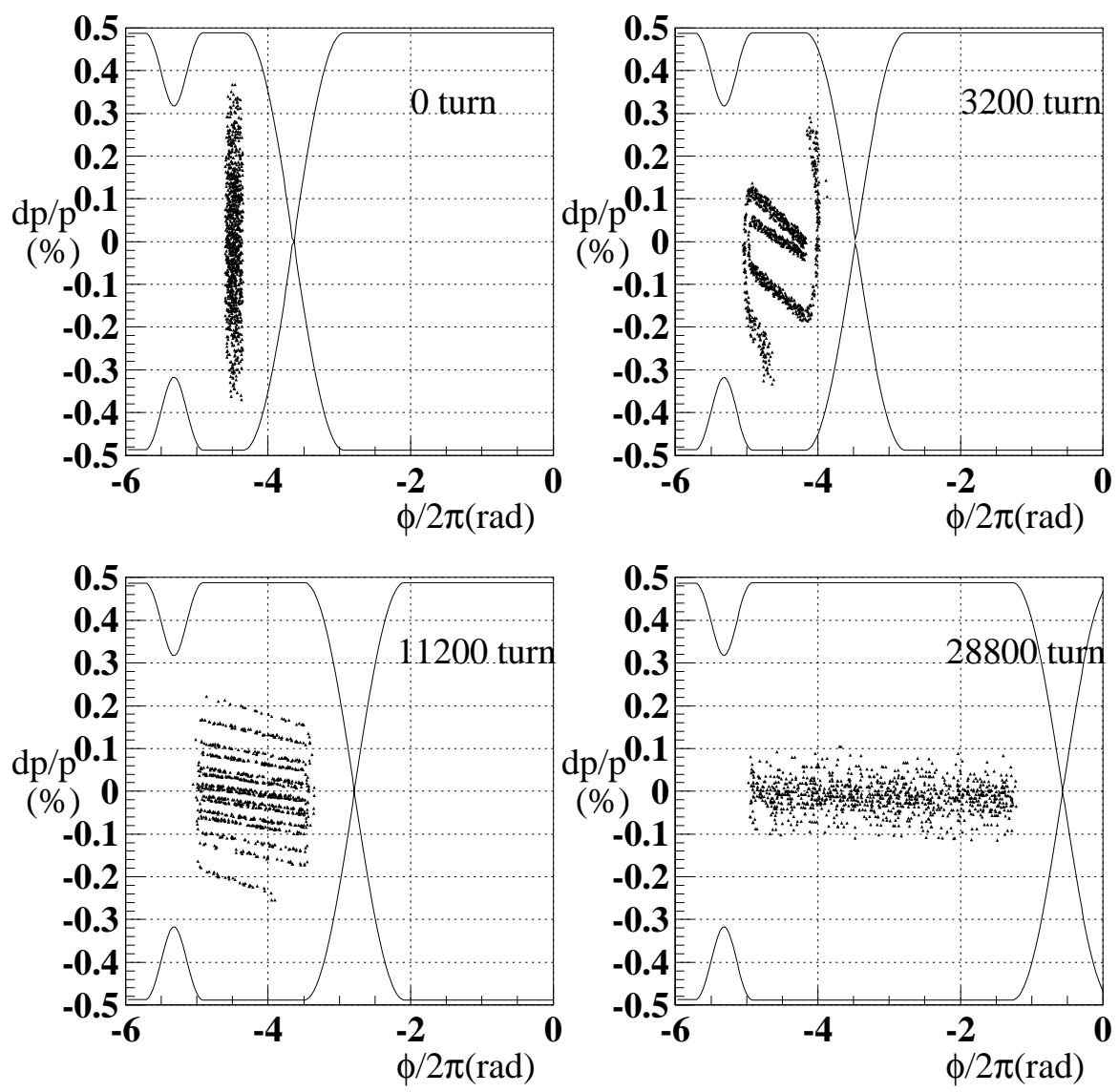

FIG. 12. Phase-space configurations during the first adiabatic debunching. The solid lines are the separatrix. The horizontal and vertical coordinates are the rf phase and momentum spread, respectively. A phase of $12 \pi$ corresponds to one revolution.

for the barriers. The width of the moving barrier (MA cavity) and the fixed barrier (ferrite cavity) were fixed at 667 and $385 \mathrm{~ns}$, respectively. The beam-loading and space-charge effects were not included in the simulation. The only longitudinal-mismatch effect was estimated in this subsection. The simulated momentum distributions after sweeping were least-square fitted with a Gaussian function, and a $d p / p$ of $\pm 2 \sigma$ was adopted. The longitudinal emittances were derived from the momentum spread multiplied by the bunch width, which are given by $\varepsilon_{l} \simeq \Delta E \times \Delta t$.

Figure 12 shows the longitudinal phase spaces during adiabatic debunching of the first bunch. The emittance increased from 2.9 to $7.3 \mathrm{eV} \mathrm{s}$ in this simulation. The blowup factor in this case was 2.5 , which is almost consistent with the ratio of the initial phase gap to the matched bucket width, which equals $99^{\circ} / 45^{\circ}$. Here, a matched bucket width of $45^{\circ}$ was given by the simulation. The longitudinal phase space at every injection is shown in Fig. 13. Some particles escape over the fixed barrier wall after the third injection, which is due to emittance growth caused by a mismatch. The longitudinal emittance finally becomes $46 \mathrm{eV} \mathrm{s}$ after five-bunch transfers with BB. The blowup factor, including the longitudinal mis- match, is 3.2 , which is in agreement with the experimental result of 2.9 .

\section{B. Potential distortion}

The potential distortion was caused by beam loading of the ferrite cavity and the overshooting voltage of the MA cavity, both of which might prevent the beam from debunching smoothly. Figure 14 shows the measured gap voltages in this BB experiment. The ringing voltage makes minibuckets and the overshooting voltage makes a bump in the longitudinal phase space, where some particles are trapped. In order to estimate the potential distortion effect, simulations of five-bunch transfers were carried out, which took account of the beam loading and the overshooting voltage in addition to any longitudinal mismatch. As for the overshooting voltage, the measured gap voltage of the MA cavity was used.

The blowup factor of the longitudinal emittance after five-bunch transfers is given in Table IV. The emittance growth was mainly caused by a longitudinal mismatch. It has been found that the contribution from the potential distortion is small. The reasons are considered to be as follows. The potential distortion induces modulation in 

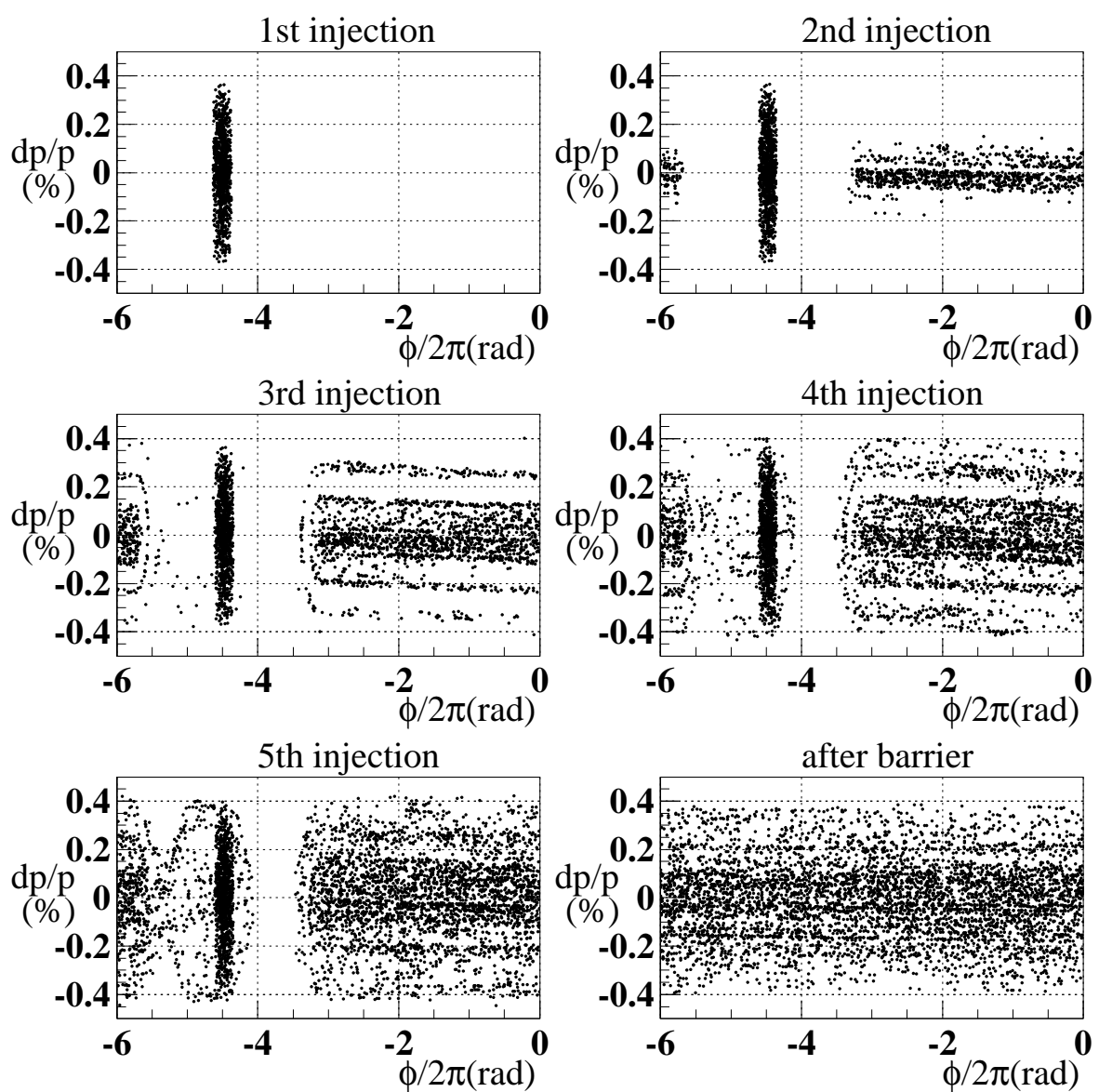

FIG. 13. Phase-space configurations at every injection. The horizontal and vertical coordinates are the rf phase and momentum spread, respectively. The initial bunch width and the momentum spread are $24^{\circ}$ and \pm 0.0038 , respectively. The blowup factor of the longitudinal emittance after five-bunch transfers is 3.2 , which is consistent with the experimental result.

the potential well. If it is small compared with the bucket height, only those particles with a smaller momentum spread are affected. In multiple transfers, the effect decreases because the momentum spread becomes larger. Further, the overshooting voltage deepens the potential well and makes a bump, where some particles are captured. It does not contribute to the growth, however, because the particles are released due to adiabatic turning off during the merging process.

In an experiment involving five-bunch transfers, a longitudinal mismatch was the main cause of emittance growth. In the case of single-bunch transfer, since the mismatch can be minimized it is possible for a potential distortion to be observed. In the adiabatic debunching process of a single bunch, a potential distortion induces growth of the momentum spread. A simulation for singlebunch transfer was performed which included varying the sweep speed of the moving barrier under the same condition as that in the experiment. Figure 15 shows the simulation results. The longitudinal phase spaces during adiabatic debunching can be seen in Fig. 16.
The results, including the beam loading, agree with the experimental results, where the minimized value of the momentum spread is consistent with the bucket height formed by the ringing voltage. The beam loading caused a growth of the momentum spread in the experiment. One can see the minibunching beam in Fig. 16(c), while the beam is compressed by the overshooting voltage and the bunching factor becomes worse in Fig. 16(e). It is desirable that such a system which can be used to compensate beam loading is prepared for the ferrite cavity. The overshooting voltage of the MA cavity is due to an imperfect waveform of the drive signal, which could be canceled by optimizing the waveform.

Moreover, the simulation results show that a sweep time slower than $100 \mathrm{~ms}$ satisfies the adiabatic condition, which is consistent with the experimental results, where the drift rate of the beam is $6.3 \times 10^{2}(\mathrm{rad} / \mathrm{s})$ and the sweep time of $100 \mathrm{~ms}$ corresponds to a sweep speed of $4.2 \times 10(\mathrm{rad} / \mathrm{s})$. It is found that a sweep speed as slow as about one-tenth of the drift rate is necessary to satisfy the adiabatic condition. 

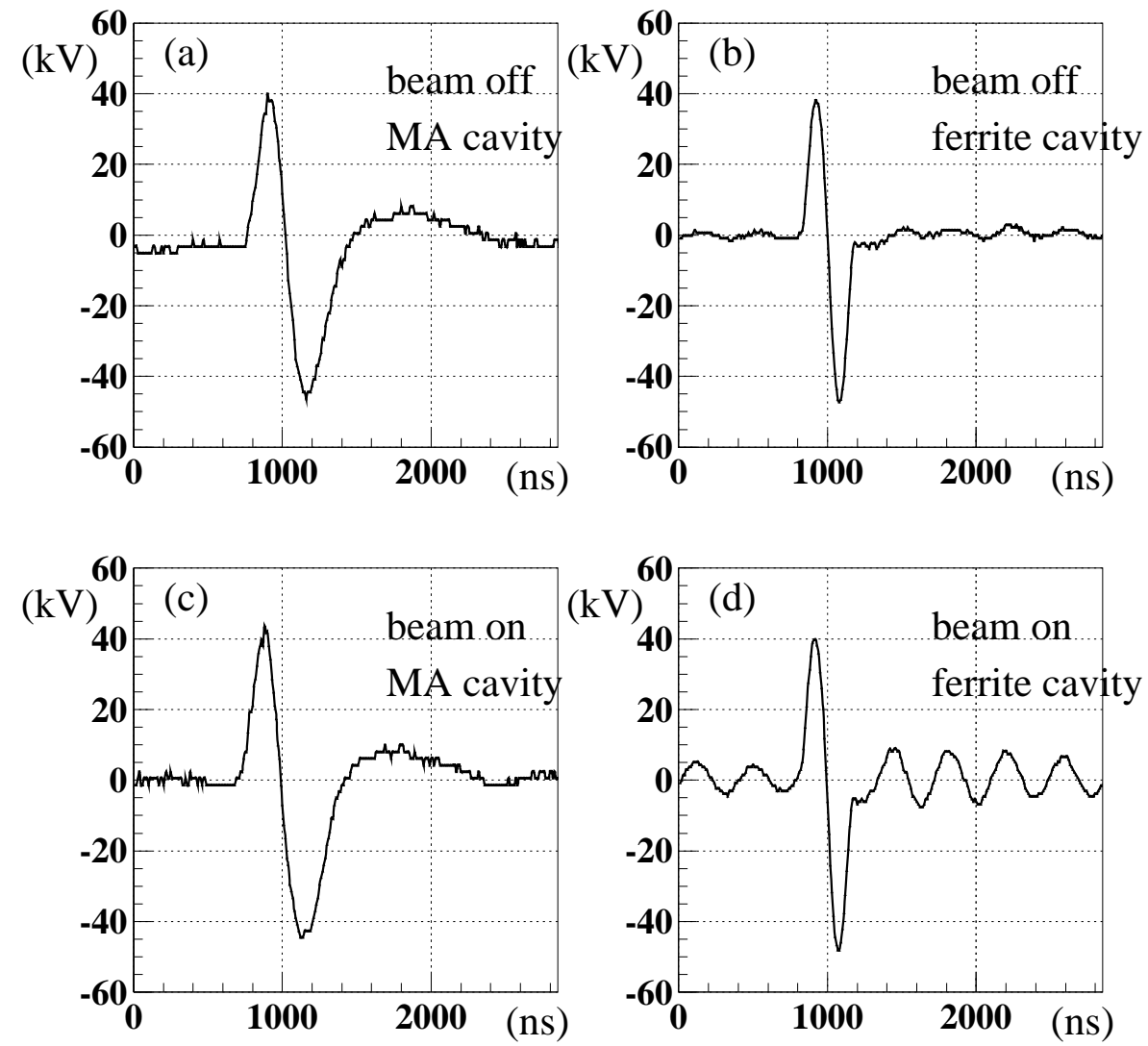

FIG. 14. Gap voltages: (a),(c) MA cavity; (b),(d) ferrite cavity. (a),(b) without beam, (c),(d) with beam. The horizontal and vertical coordinates are time and voltage per gap, respectively. The beam loading of the MA cavity was compensated by feed forward. The beam intensity was $8.7 \times 10^{12}$ protons/bunch.

\section{Prospect}

We have found that a longitudinal mismatch caused mainly a longitudinal emittance growth in the present experiment. The longitudinal mismatch was caused by a wider pulse width of the injection kicker than the bunch width. In order to reduce the longitudinal emittance growth, a scheme using barriers having widths equal to the kicker pulse may be possible. The ferrite cavity can generate such barriers if the resonant frequency decreases by adding capacitance to the gaps. Further, the beamloading compensation system and any system to carry out adiabatic turning off are desired for the ferrite cavity.

The width of the barrier generated by the MA cavity is adjustable due to its broadband characteristics. Adiabatic

TABLE IV. Blowup factor of the longitudinal emittance.

\begin{tabular}{cc}
\hline \hline Effect & Blowup factor \\
\hline Longitudinal mismatch & 3.2 \\
+ overshooting voltage & 3.1 \\
$\quad+$ beam loading & 3.6 \\
Experimental results & 2.9 \\
\hline \hline
\end{tabular}

turning off is also possible. Moreover, beam loading is no problem for the MA cavity, as described above, using feed-forward beam-loading compensation. One can expect that the emittance growth can be decreased by using only the MA cavity, although larger power tetrodes are necessary due to plate dissipation under practical operation. In order to clarify this, a simulation of fivebunch transfers was performed while assuming that the MA cavity generates both fixed and moving barriers. In this simulation, the width of the barrier was fixed at $770 \mathrm{~ns}$, which is equal to the pulse width of the injection kicker. Two single sine waves were used for the waveforms. The initial longitudinal emittance is $2.4 \mathrm{eV} \mathrm{s}$, which is a typical emittance at a beam intensity of $8 \times 10^{12}$ protons in the $\mathrm{BB}$ experiment.

The total emittance becomes $18 \mathrm{eV} \mathrm{s}$ after the accumulation of five bunches, of which the blowup factor due to a longitudinal mismatch becomes 1.5. This value is smaller than the experimental result of 2.9. Moreover, the mismatch can be further reduced by adjusting the bunch width with the quadrupole bunch-shape oscillation at the booster. The longitudinal phase spaces are shown in Fig. 17. There are no particles that escape from the barrier walls. One can see that the rf bucket is not full 


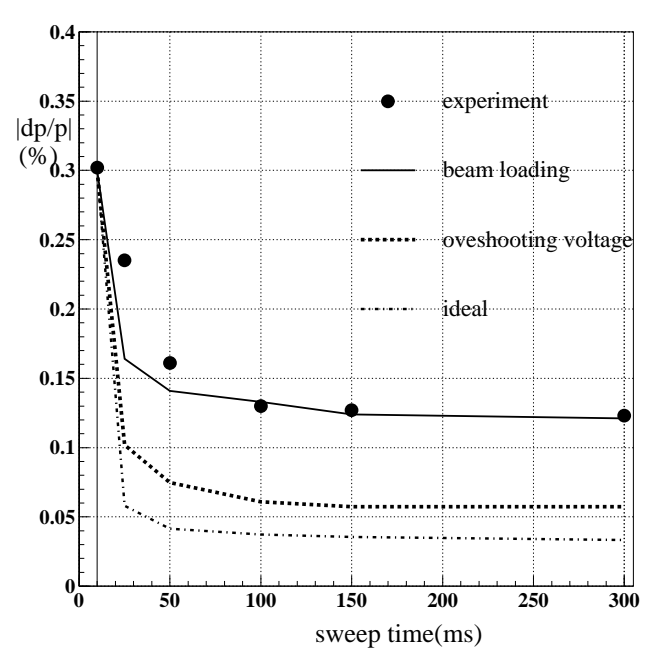

FIG. 15. Simulation results about adiabatic debunching. The horizontal and vertical coordinates are the sweep time and momentum spread, respectively. The initial bunch width and momentum spread were $24^{\circ}$ and \pm 0.003 , respectively. The beam intensity was $7.0 \times 10^{12}$ protons/bunch. The longitudinal mismatch was minimized. The initial momentum spreads are shown on the solid line. The case without any potential distortion is presented as "ideal." The results, including beam loading, agree with the experimental results. after an accumulation of $4 \times 10^{13}$ protons in Fig. 17. It is possible to afford to accumulate more bunches. Further, since beam loading is negligible for the MA cavity, the intensity per bunch can be increased.

\section{CONCLUSION}

An MA-loaded cavity was developed for the barrier bucket experiment. It could generate a single sine wave with less rf power because of the low $Q$ value. Beamloading compensation was successfully performed. Its operational flexibility and stability were suitable for the barrier bucket.

A barrier bucket experiment was carried out at the AGS. A barrier system using two dedicated barrier cavities was established. Five bunches were accumulated with the barriers. A total of $3 \times 10^{13}$ protons were rebunched and accelerated by accelerating cavities having a harmonic number of 6 . The slow beam loss during the accumulation was negligible. It is found that a barrier bucket scheme is efficient for accumulating a beam in a proton synchrotron.

The longitudinal emittance growth was observed during accumulation by the barriers. The simulation results
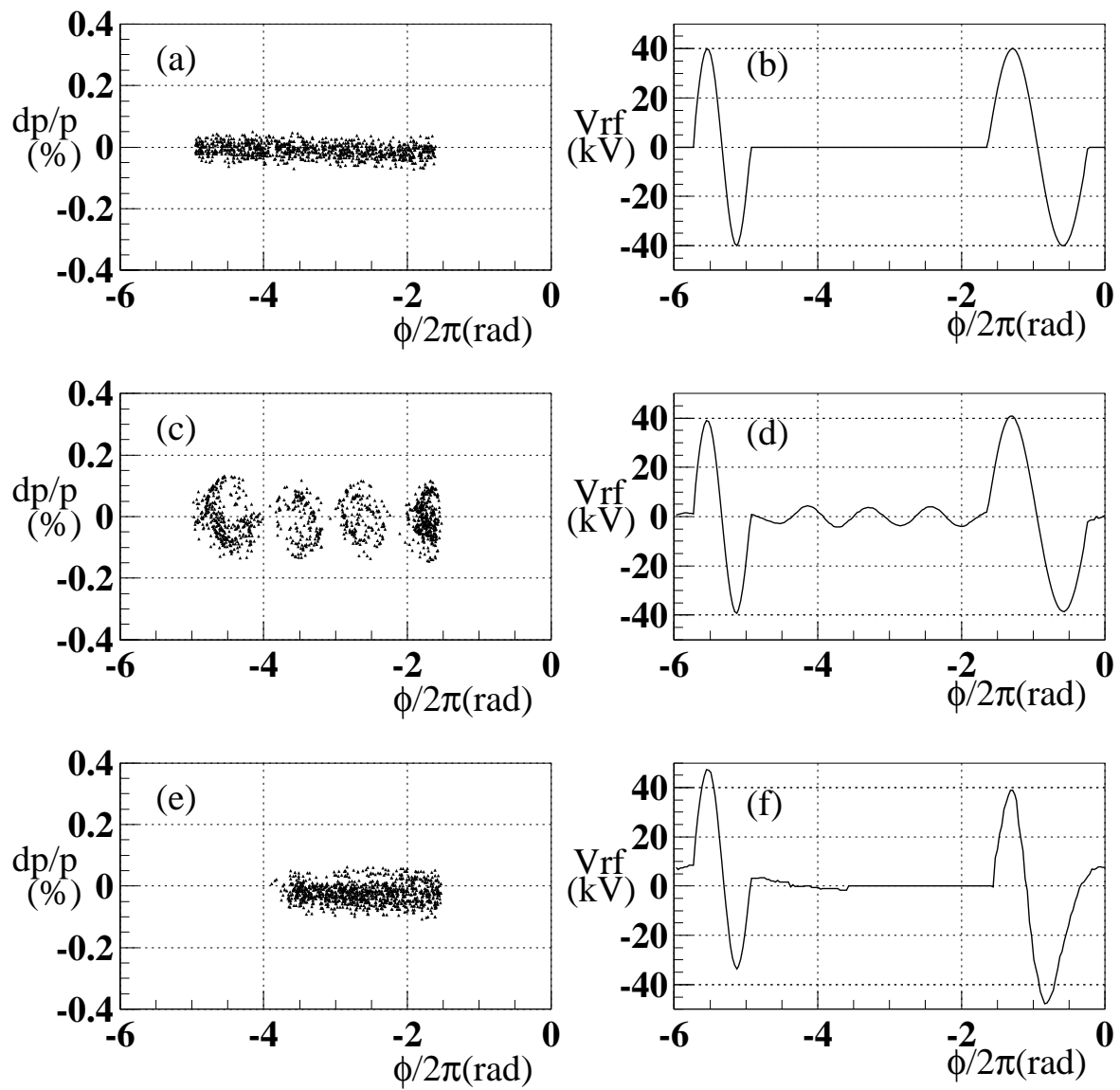

FIG. 16. Phase-space configurations: (a),(c),(e) momentum spreads; (b),(d),(f) rf voltages. (a),(b) ideal state; (c),(d) with the beam loading by the ferrite cavity; (e),(f) with the overshooting voltage of the MA cavity. The sweep time is $100 \mathrm{~ms}$. The horizontal axis is rf phase. The beam intensity is $7 \times 10^{12}$ protons/bunch. 

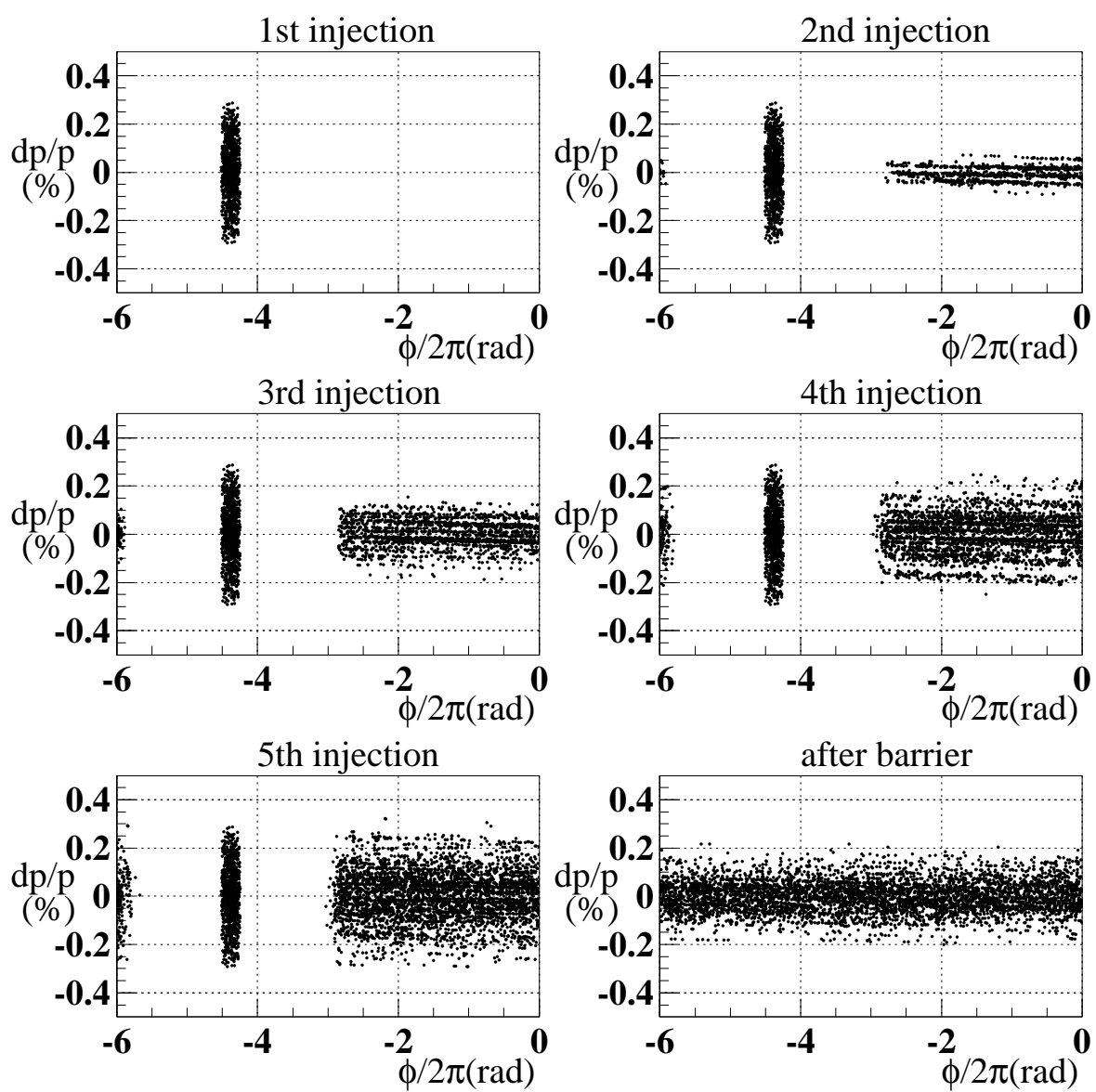

FIG. 17. Phase-space configurations at every injection. The horizontal and vertical coordinates are the rf phase and momentum spread. The intensity of one bunch is set at $8 \times 10^{12}$ protons. The bunch width and initial momentum spread are set at $24^{\circ}$ and \pm 0.003 , respectively. The phase gap between the barriers is $97^{\circ}$, which is equal to the pulse width of the injection kicker.

showed that the main cause is a longitudinal mismatch due to a wider phase gap than the bunch width. The result of five-bunch transfers was almost consistent with the experimental result. It was found that the potential distortion caused by beam loading of the ferrite cavity and the overshooting voltage of the MA cavity prevents the beam from debunching smoothly. The developments of an injection kicker and a beam-loading compensation system for the ferrite cavity are desirable. The overshooting voltage could be canceled by optimizing the drive signal. It is possible to reduce the emittance growth if such improvements are performed, which would help the beam intensity to be increased.

\section{ACKNOWLEDGMENTS}

We would like to thank members of the KEK-Tanashi $\mathrm{RF}$ group for the development of the MA cavity. We are grateful to members of the AGS RF group for much help. We would like to thank the AGS operators for their skillful tuning and cooperation. This work was performed under the auspices of the U.S. Department of Energy.
[1] J. Griffin et al., IEEE Trans. Nucl. Sci. 30, 3502-3504 (1983).

[2] M. Blaskiewicz and J.M. Brennan, in Proceedings of the 5th European Particle Accelerator Conference, Barcelona, Spain, 1996 (Institute of Physics, Bristol, UK, 1996), pp. 2373-2376.

[3] S. Y. Lee and K. Y. Ng, Phys. Rev. E 55, 5992-6001 (1998).

[4] M. Fujieda et al., in Proceedings of the 1999 Particle Accelerator Conference, New York (IEEE, Piscataway, NJ, 1999), pp. 857-859.

[5] M. Blaskiewicz et al., in Proceedings of the 1999 Particle Accelerator Conference, New York (Ref. [4]), pp. 22802282.

[6] L. Ahrens et al., in Proceedings of the 1999 Particle Accelerator Conference, New York (Ref. [4]), pp. 614616.

[7] M. Fujieda et al., in Proceedings of the First Asian Particle Accelerator Conference, Tsukuba, Japan, 1998 (KEK, Tsukuba, 1990), pp. 408-410.

[8] C. Ohmori et al., in Proceedings of the 1999 Particle Accelerator Conference, New York (Ref. [4]), pp. 413-417.

[9] Y. Mori et al., in Proceedings of the 6th European Particle Accelerator Conference, Stockholm, 1998 (Institute of Physics, Bristol, UK, 1998), pp. 299-301. 
[10] K.Y. Ng and Z.B. Qian, in Proceedings of the 1999 Particle Accelerator Conference, New York (Ref. [4]), pp. 872-874.

[11] P. Barrat et al., in Proceedings of the 2nd European Particle Accelerator Conference, Nice, 1990 (Editions
Frontières, Gif-sur-Yvette, 1990), pp. 949-951.

[12] F. Blask and R. Garoby, in Proceedings of the 1991 Particle Accelerator Conference, San Francisco (IEEE, Piscataway, NJ, 1991), pp. 1398-1400.

[13] Y. Hashimoto et al., KEK Report No. 98-104, 1998. 